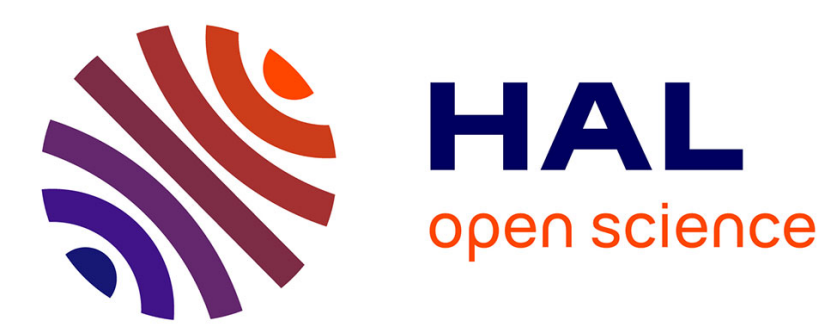

\title{
Integrated production scheduling and delivery routing: complexity results and column generation
}

\author{
Azeddine Cheref, Christian Artigues, Jean-Charles Billaut, Sandra Ulrich
}

Ngueveu

\section{- To cite this version:}

Azeddine Cheref, Christian Artigues, Jean-Charles Billaut, Sandra Ulrich Ngueveu. Integrated production scheduling and delivery routing: complexity results and column generation. 4th International Symposium on Combinatorial Optimization (ISCO'2016), May 2016, Vietri sur Mare, Italy. hal01304786

\section{HAL Id: hal-01304786 \\ https://hal.science/hal-01304786}

Submitted on 26 Aug 2016

HAL is a multi-disciplinary open access archive for the deposit and dissemination of scientific research documents, whether they are published or not. The documents may come from teaching and research institutions in France or abroad, or from public or private research centers.
L'archive ouverte pluridisciplinaire HAL, est destinée au dépôt et à la diffusion de documents scientifiques de niveau recherche, publiés ou non, émanant des établissements d'enseignement et de recherche français ou étrangers, des laboratoires publics ou privés. 


\title{
Integrated production scheduling and delivery routing: complexity results and column generation
}

\author{
Azeddine Cheref ${ }^{1,2}$, Christian Artigues ${ }^{1}$, Jean-Charles Billaut ${ }^{2}$, and \\ Sandra Ulrich Ngueveu ${ }^{1}$ \\ ${ }^{1}$ LAAS-CNRS, Université de Toulouse, CNRS, INP, Toulouse, France \\ ${ }^{2}$ Laboratoire d'Informatique de l'Université de Tours, \\ 64 avenue jean Portalis, 37200 Tours, France \\ \{artigues, cheref, ngueveu\}@laas.fr \\ jean-charles.billaut@univ-tours.fr
}

\begin{abstract}
In this paper, we study an integrated production scheduling and delivery routing problem. The manufacturer has to schedule a set of jobs on a single machine without preemption and to deliver them to multiple customers. A single vehicle with limited capacity is used for the delivery. For each job are associated: a processing time, a size and a specific customer location. The problem consists then to determine the production sequence, to constitute batches and to find the best delivery sequence for each batch. The objectives of the proposed problems are to find a coordinated production and a delivery schedule that minimizes the total completion time (makespan) or the sum of the delivery times of the products. We present complexity results for particular cases and a column generation scheme to solve a relaxed version of the problem, leading to a lower bound of high quality. Some computational results show the good performances of the method.
\end{abstract}

Keywords: Integrated production and distribution; Complexity; Column generation

\section{Introduction}

This paper considers an integrated model of scheduling and delivery, where jobs are scheduled on a single machine and finished products are delivered from the manufacturer to multiple customer locations. The relationship between production and distribution being strong, an increasing amount of research has been devoted to this field during the last years. The problem has been largely analysed and reviewed in [4], where the author proposes a classification scheme for a variety of issues reflected by these models.

In this paper, jobs are scheduled on a single machine and preemption is not allowed. Different processing times, sizes and delivery destinations are associated to the jobs. Distribution is performed by a single vehicle with a limited capacity 
and can be seen as a variant of the multitrip vehicle routing problem [15], in which deliveries are ensured by a single vehicle and with a constrained batching phase. Delivery costs are not taken into account in this paper but the total time required to complete the production and the delivery of the products and the total delivery time are both meaningful indicators of the overall efficiency of the delivery process. Therefore, the objectives of the proposed problems are to find a coordinated production and delivery schedule that minimize the total completion time (makespan) or the sum of the delivery times of the products.

We review below a few relevant papers.

In [13], a similar model is considered but the size of jobs is not included and the authors propose a polynomial time algorithm in the case of a fixed number of distinct destinations. In [6], the authors consider the problem of minimizing the makespan on a single machine scheduling problem with a unique capacitated vehicle and a no wait constraint. No wait constraints implies that the batch must be delivered at its completion time. In [8], an heuristic method is proposed for minimizing the makespan when lifespan constraints are introduced for the products. Most of the models presented in the literature explicitly take into account transportation times to reach the customer's location, but there are no proper routing decisions, since the number of distinct customers is typically very small. Hence, the focus of the analysis is often on scheduling and batching. In [10], the authors consider the problem in which the delivery dates are fixed in advance and in [5], there are various destinations but a batch must contain jobs of the same destination. Complexity results are given by [3] for the problem with a single vehicle, a storage area and one or two customers. In [12], the authors minimize the makespan for the one machine scheduling problem with pickup and delivery in which a single vehicle travels between the machines and the warehouse, whereas in [17], the authors study a similar problem in which three different locations and two vehicles are considered. The first vehicle transports unprocessed jobs between the warehouse and the factory and the second one transports finished jobs between the factory and the customer. Some models in the literature treat a coordinating problem in which the customer sequence is fixed. For example, in [1] and [16], the authors minimize the total satisfied demand in a single round trip, the authors consider that the products expire in a constant time after their completion time and a time window delivery for each product.

The problem is formally defined in section 2 . We present in Section 3 some complexity results for particular cases and in section 4 a column generation scheme to solve a relaxed version of the problem, leading to a lower bound of high quality. Computational results are given in Section 5 .

\section{Problem definition and notations}

We consider a set of $n$ jobs $J=\left\{J_{1}, J_{2}, \ldots, J_{n}\right\}$ to be processed on a single machine and delivered to a set of $n$ corresponding customers. Each job $J_{j}$, $j=1, \ldots, n$, requires a certain processing time $p_{j}$. Delivery is performed by a 
single vehicle with capacity $c$. As mentioned before, there is a set of $n$ customer locations and each job $J_{j}, j=1, \ldots, n$, is additionally characterized by its location $j$ and its size $s_{j}$ where $0 \leq s_{j} \leq c$. We denote by $t_{i j}$ the transportation time from location $i$ to location $j$ and $D_{j}$ the arrival time (decision variable) to the location $j$,i.e. the delivery time of $J_{j}$. We use $M$ to denote the machine and, by analogy with vehicle routing problems, we refer to the machine location as the depot.

The vehicle loads a certain number of jobs which have been processed and starts the round trip to deliver them at their respective locations. The set of jobs delivered during a single round trip is called a batch. The problem is then to determine the scheduling sequence, cluster the jobs into batches and determine the best route for each batch. Using the notation introduced by [11], the general problem considered here with one machine, several customers, one vehicle and a limited capacity is denoted by $1 \rightarrow D, k \geq 1|v=1, c| D_{\max }$ for the makespan objective and $1 \rightarrow D, k \geq 1|v=1, c| \sum D_{j}$ for the total completion time $(1 \rightarrow D$ means "one machine to delivery", $k$ is the number of customers, $v$ is the number of vehicles, $c$ indicates that a capacity is considered). An illustration for the problem with $n=7$ jobs and $n$ customers is given in Fig. 1 .

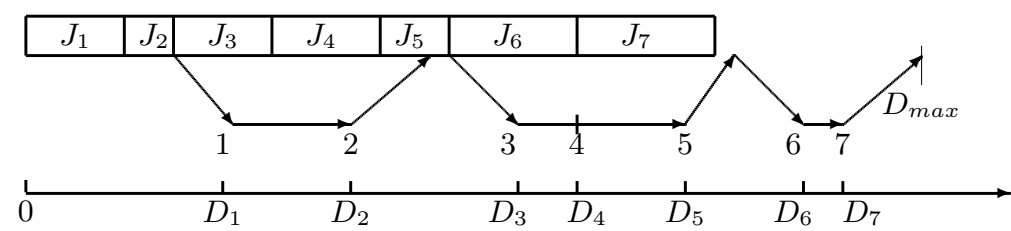

Fig. 1. An illustration for problems $1 \rightarrow D, k \geq 1|v=1, c| D_{\max }$ or $\sum D_{j}$

We first discuss the complexity of special cases of the problem. Then we propose extended formulations and a column generation framework for the general case.

\section{Particular cases}

In this section, we consider two special cases of the problem for both objectives functions. We give complexity results for the single customer case and some remarks for the fixed-batches case.

\subsection{One customer case}

In [3], the authors prove that the problem $1 \rightarrow D, k=1|v=1, c| D_{\max }$ is equivalent to the NP-hard Bin Packing problem when the processing times $p_{j}=0$ for all $j$. Note that this reasoning becomes invalid for the total delivery time objective. However, we prove that problem $1 \rightarrow D, k=1|v=1, c| \sum_{j} D_{j}$ is 
strongly NP-hard by reduction from 3-PARTITION problem. For our purpose, we introduce the 3-PARTITION problem.

3-PARTITION. Given $3 h$ integers $a_{1}, \ldots, a_{3 h}$, so that $\sum a_{i}=h b$, and such that $b / 4<a_{i}<b / 2$ for all $i$, is it possible to partition them into $h$ disjoint sets each summing up to $b$ ?

Theorem 1. Problem $1 \rightarrow D, k=1|v=1, c=z| \sum_{j} D_{j}$ is NP-hard in the strong sense.

Proof. Given a 3-PARTITION instance, we construct an instance for our problem as follows:

$$
\begin{array}{r}
n=3 h \text { jobs, } c=b, t_{M 1}=t_{1 M}=t \text { and } t>0 \\
\text { For each job } J_{j}: p_{j}=0, s_{j}=a_{j} \\
\text { Sum of the delivery times } y=3 t h^{2}
\end{array}
$$

From there, the problem consists in determining whether a solution exists such that $\sum_{j} D_{j} \leq y$.

$\rightarrow$ If there is 3-PARTITION, then there exists a feasible schedule to our problem with $\sum_{j} D_{j} \leq y$. Let $H_{1}, H_{2}, \ldots, H_{h}$ be a solution of 3-PARTITION. Then, we construct a schedule to our problem by setting each batch $b_{i}$ to the triple $H_{i}$. The vehicle starts the tour at time zero, delivers the first three jobs at time $t$ and is back at the depot at $2 t$. Since the processing times are equal to zero, the vehicle restarts immediately and the second batch is delivered at time $3 t$. Following this reasoning (see Fig. 2), a batch $b_{i}$ is delivered at time $(2 i-1) t$ and the total delivery time $\sum_{j} D_{j}=3 \sum_{i=1}^{h}(2 i-1) t=y$.

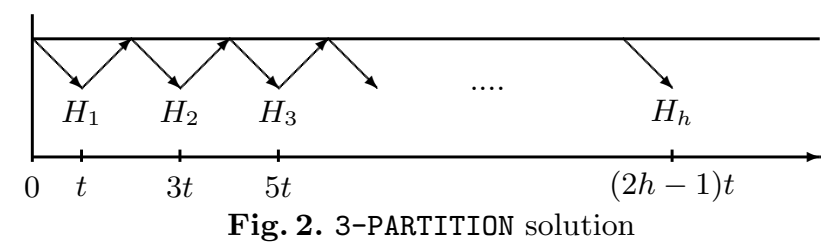

$\leftarrow$ Suppose that a schedule $S$ for our problem exists in which $\sum_{j} D_{j} \leq y$. According to the generated instance, the number of batches in $S$ cannot be smaller than $h$, the number of jobs in each batch cannot exceed 3 and the vehicle is never idle at the depot in an optimal solution. We denote by $h^{\prime}$ the number of batches in $S$. Firstly, we suppose that $h^{\prime}=h$ and denote by $S_{1}$ the corresponding schedule. This implies that each batch $b_{1}, b_{2}, \ldots, b_{h}$ of $S_{1}$ contains exactly three jobs and for each batch $\sum_{j \in b_{i}} s_{j}=b$. Thus, $b_{1}, \ldots, b_{h}$ define a solution of the 3-PARTITION problem. Suppose now that there exists a schedule $S_{2}$ for which $\sum_{j} D_{j} \leq y$ and $h^{\prime}>h$. Let $n_{1}$ the number of jobs in $b_{1}, \ldots, b_{h}$ and $n_{2}$ the number of jobs in $b_{h+1}, \ldots, b_{h^{\prime}}$. We denote by $\sigma_{j}^{S_{1}}$ and $\sigma_{j}^{S_{2}}$ the jobs scheduled at position $j$ in the schedule $S_{1}$ and $S_{2}$ respectively. Due to the fact that each 
batch in $S_{1}$ contains three jobs, one can see that $\sum_{j=1}^{n_{1}} D_{\sigma_{j}^{S_{2}}} \geq \sum_{j=1}^{n_{1}} D_{\sigma_{j}^{S_{1}}}$. In the schedule $S_{2}$, the remaining $n_{2}$ jobs are delivered after the time $(2 h-1) t$ which represents the delivery time of $J_{\sigma_{n_{1}}^{S_{2}}}$ and the last job $J_{\sigma_{n}^{S_{1}}}$ in $S_{1}$. So, $\sum_{j=n_{1}+1}^{n} D_{\sigma_{j}^{S_{2}}}>\sum_{j=n_{1}+1}^{n} D_{\sigma_{j}^{S_{1}}}$ which implies $\sum_{j} D_{j}>y$ on the solution $S_{2}$.

Remark 1. Problem $1 \rightarrow D, k=1|v=1, c| \sum_{j} D_{j}$ is polynomially solvable when all jobs have the same size [13]. The authors propose a polynomial time algorithm with a complexity in $O\left(n^{2}\right)$ to solve the problem.

\subsection{Fixed-batch case}

In this case, we consider that the jobs are already clustered into batches and that, for each one, the delivery route is known. We consider below the makespan criterion and the sum of delivery times criterion.

\section{$1 \rightarrow D, k=1 \mid v=1, c$, fixed - batches $\mid D_{\max }$ problem}

Proposition 1. Problem $1 \rightarrow D, k \geq 1 \mid v=1, c=z$, fixed-batches $\mid D_{\max }$ is polynomially solvable.

If we consider a batch as a job, this problem becomes equivalent to the wellknown polynomial two-machine flow shop problem with makespan criterion [9]. In the resulting problem, we consider the duration of the batch on the machine as the processing time of the corresponding job on the first machine and the duration of the route of the batch as the processing time on the second machine.

$1 \rightarrow D, k=1 \mid v=1, c$, fixed - batches $\mid \sum_{j} D_{j}$ problem

Proposition 2. Problem $1 \rightarrow D, k \geq 1 \mid v=1, c=z$, fixed-batches $\mid \sum_{j} D_{j}$ is NP-hard.

We consider the case in which each batch contains exactly one job and we denote by $C_{j}^{\prime}$ the time at which the vehicle is back at the depot after the delivery of job $J_{j}$. The delivery time $D_{j}$ of a job $J_{j}$ is then $D_{j}=C_{j}^{\prime}-t_{j M}$ and $\sum_{j=1}^{n} D_{j}=$ $\sum_{j=1}^{n} C_{j}^{\prime}-\sum_{j=1}^{n} t_{j M}$, with $\sum_{j}^{n} t_{j M}$ a constant. This problem is equivalent to solving the NP-hard two-machine flow shop problem with the sum of completion times criterion [7], in which the processing times of a job $J_{j}$ on the first machine is equal to $p_{j}$, equal to $t_{M j}+t_{j M}$ on the second machine and a completion time on the second machine equal to $C_{j}^{\prime}$.

\section{General case}

For the general case, we first establish the following fundamental dominance property. As there are no release dates for the jobs, from any solution, the machine sequence can obviously be reordered according to the routing sequence 
and the jobs can be scheduled at the earliest without increasing the objective function for both the $D_{\max }$ and $\sum_{j} D_{j}$ criteria. The property then follows:

Property 1. There exists an optimal solution satisfying the following conditions:

- Jobs are processed on the machine without idle time,

- Production sequence and routing sequence are the same.

This implies that when the round trip of a batch is given, the sequence on the machine can be deduced. For both objective functions, one can see that the optimal solution of the problem can be obtained by combining batches and the problems are able to be modeled as a set covering problem. Hence, extended formulations and column generation approaches can be considered for both problems. Note that if there was no machine scheduling phase, the problem would resort to the multi-trip travelling salesman problem. Below, we detail these approaches for each criterion. Note that column generation and branch-and-price are techniques of choice for the related multi-trip vehicle routing problem [2],[15]. However, we have in our case a single vehicle and a preliminary constrained batching phase due to the machine sequencing sub-problem. It is thus relevant to wonder whether a column generation approach can still be successfully applied or not.

\section{1 $1 \rightarrow D, k \geq 1|v=1, c| D_{\max }$ problem}

We introduce in this section a set covering formulation for the master problem. A column represents a batch and its position on the delivery sequence. The set of feasible batches is denoted as $\beta$. For each batch $b \in \beta$, its duration on the machine and its round trip duration are known. We denote by $P_{b, 1}=\sum_{j \in b} p_{j}$ the duration of the batch $b$ on the machine and, $P_{b, 2}$ the duration of the round trip that delivers the batch $b$. Since the jobs contained in a batch are known, $a_{i, b}$ takes the value 1 if the job $J_{i}$ is in the batch $b$ and 0 otherwise. A unique set of variables $x_{b, k}$ is used to minimize the $D_{\max }$ objective. Variables $x_{b, k} \in\{0,1\}$ 
indicates if the batch $b$ at the position $k$ is selected.

$$
\begin{array}{cr}
\min D_{\text {max }} & \\
D_{\text {max }} \geq \sum_{k=1}^{l} \sum_{b \in \beta} P_{b, 1} x_{b, k}+\sum_{k=l}^{n} \sum_{b \in \beta} P_{b, 2} x_{b, k}, & \forall l \in\{1, \ldots, n\} \\
\sum_{b \in \beta}\left(a_{i, b} \sum_{k=1}^{n} x_{b, k}\right)=1, & \forall k \in\{1, \ldots, n\} \\
\sum_{b \in \beta} x_{b, k} \geq \sum_{b \in \beta} x_{b, k+1}, & \forall i \in\{1, \ldots, n\} \\
\sum_{b \in \beta} x_{b, k}=1, & \forall k \in\{1, \ldots, n-1\} \\
x_{b, k} \in\{0,1\} & \forall k \in\{1, \ldots, n\}, \forall b \in \beta
\end{array}
$$

The first set of constraints (2) is equivalent to the fixed-batches case presented above. It ensures that the processing of a batch on the machine starts after the completion of the previous one and that the vehicle starts the delivery of a batch after its completion on the machine, and the end of the previous tour. Constraints (3) state that a position can contain at most one batch and constraints (4) ensure that each job is contained in exactly one selected batch. Constraints (5) are symmetry breaking constraints that enforce that all selected columns (batches) appear consecutively at the first positions. Constraints (6) sets a minimum number of batches using a lower bound $\delta$ of the number of round trips to deliver all the jobs. To obtain $\delta$, we use the First Fit Decreasing rule which is a $3 / 2$ approximation for the Bin Packing problem. Let $\tau$ the number of bins obtained by the FFD algorithm. A lower bound of the minimum number of batches $\delta$ is then equal to $2 / 3 \tau$.

Following a standard column generation scheme, the master problem is restricted to a subset of variables (columns) $\tilde{\beta} \subseteq \beta$ and a pricing problem is needed to find new non basic variables that can improve the solution for the LP relaxation of model (1-7).

The pricing problem This sub-problem searches for an element of $\beta \backslash \tilde{\beta}$ such that the reduced cost of the new column is negative. We denote by $\bar{c}_{b, k}$ the reduced cost of $x_{b, k}$ and, one has:

$$
\bar{c}_{b, k} \leq 0 \Leftrightarrow \sum_{i=1}^{n} \underbrace{\frac{p_{i} \sum_{l=k}^{n} \alpha_{l}-\gamma_{i}}{\sum_{l=1}^{k} \alpha_{l}}}_{l_{i}} a_{i, b}+P_{b, 2}<\underbrace{\frac{\beta_{k}-\sigma_{k-1}+\sigma_{k}+\xi_{k}}{\sum_{l=1}^{k} \alpha_{l}}}_{r_{k}}
$$

where $\alpha_{l}, \beta_{k}, \gamma_{i}, \sigma_{k}$ and $\xi_{k}$ denote the dual values associated to the constraints $(2),(3),(4),(5),(6)$ respectively. Note that the dual values $\xi_{k}$ exist only for $k \leq \delta, \sigma_{k-1}$ for $k \geq 2$ and $\sigma_{k}$ for $k \leq n-1$. 
We define an auxiliary directed graph $G=(V, A)$ in which $V=N \cup\left\{v_{s}, v_{d}\right\}$ where nodes $N=\left\{v_{1}, \ldots, v_{n}\right\}$ represent the locations $1, \ldots, n$ and nodes $\left\{v_{s}, v_{d}\right\}$ the duplicated depot. The set of arcs is $A=\left\{\left(v_{i}, v_{j}\right) \mid v_{i} \in N \cup\left\{v_{s}\right\}, v_{j} \in\right.$ $\left.N \cup\left\{v_{d}\right\}, v_{i} \neq v_{j}\right\}$. Finally, the sub-problem consists in solving an elementary shortest path problem with resource constraints (ESPPRC) on graph $G$, in which the distance value $d_{i j}$ of an arc $\left(v_{i}, v_{j}\right)$ is equal to $l_{i}+t_{i j}$ for $v_{i} \in V-\left\{v_{s}\right\}$, $d_{s j}=t_{M j}$ for each arc $\left(v_{s}, v_{j}\right)$, and $d_{s d}=r_{k}$ for $\operatorname{arc}\left(v_{s}, v_{d}\right)$. The constraints concern the capacity of the vehicle. A column $x_{b k}$ corresponding to an elementary shortest path in $G$ is introduced in the restricted master problem only if the length of such path is smaller than $r_{k}$. From the resulting path and given that the jobs sequence on the machine and the delivery sequence are the same, the round trip length and the batch processing on the machine can be obtained. An exact method is used to solve the elementary shortest path problem with resource constraints. The interested reader will find more details about the used algorithm in [14].

Starting from the first position, the sub-problem searches for a new column to add by scanning all positions and stops when a column with negative reduced cost is found. The new column is then added. We propose below a heuristic to initiate the column generation process, taking account of this particularity.

Initial solution heuristic In order to accelerate the sub-problem solution phase by using a minimal number of positions, we propose the following initial solution heuristic:

(1) The first step is to assign jobs into batches according to their sizes. The First Fit Decreasing rule is used for this purpose.

(2) Given a constitution of the batches, the route is determined using the nearest neighbor search rule.

(3) As soon as the duration of the batch on the machine and the duration of the routes are known, the Johnson's rule (known to solve the two-machine flow-shop problem to optimality) is used to optimally order the batches.

\section{2 $1 \rightarrow D, k \geq 1|v=1, c| \sum_{j} D_{j}$ problem}

In this part, a formulation for the general problem with a cumulative objective function $\sum_{j} D_{j}$ is suggested. In order to obtain the delivery time of each job, the new columns of the master problem must take the departure time of a batch into account. Hence, we define binary variables $y_{b k t}$, which take the value 1 if the batch $b$ is delivered at position $k$ and starts to deliver it at time $t$ if selected, 0 otherwise. For each position $k$, the departure time is given by variable $S_{k} \geq 0$. As the considered objective needs the exact delivery time of each job, we denote by $R_{i b}$ the time between the departure time of the vehicle and the arrival time to location $i$. This value is known once a batch $b$ is given. Let $T$ be an upper bound on the latest possible departure time of the vehicle for the last batch. As 
long as the triangle inequality holds, this is given by:

$$
T=\sum_{i=1}^{n}\left(p_{i}+2 t_{0, i}\right)
$$

The other notations remain the same as those used for the formulation with the $D_{\max }$ objective function. Let $\beta^{\prime}$ denote the set of feasible batches.

$$
\begin{aligned}
& \text { Minimize } \sum_{b \in \beta^{\prime}} \sum_{k=1}^{n} \sum_{t=1}^{T} \sum_{i \in b} a_{i, b}\left(t+R_{i, b}\right) y_{b, k, t} \\
& S_{k^{\prime}} \geq \sum_{k=1}^{l} \sum_{b \in \beta^{\prime}} \sum_{t=1}^{T} P_{b, 1} y_{b, k, t}+\sum_{k=l}^{k^{\prime}-1} \sum_{b \in \beta^{\prime}} \sum_{t=1}^{T} P_{b, 2} y_{b, k, t}, \\
& \forall k^{\prime} \in\{1, \ldots, n\}, \quad \forall l \in\left\{1, \ldots, k^{\prime}\right\} \\
& \sum_{b \in \beta^{\prime}} \sum_{t=1}^{T} y_{b, k, t} \leq 1, \quad \forall k \in\{1, \ldots, n\} \\
& \sum_{b \in \beta^{\prime}} \sum_{t=1}^{T}\left(a_{i, b} \sum_{k=1}^{n} y_{b, k, t}\right) \geq 1, \quad \forall i \in\{1, \ldots, n\} \\
& \sum_{b \in \beta^{\prime}} \sum_{t=1}^{T} t y_{b, k, t} \geq S_{k}, \quad \forall k \in\{1, \ldots, n\} \\
& \sum_{b \in \beta^{\prime}} \sum_{t=1}^{T} y_{b, k, t} \geq \sum_{b \in \beta^{\prime}} \sum_{t=1}^{T} y_{b, k+1, t}, \quad \forall k \in\{1, \ldots, n-1\} \\
& \sum_{b \in \beta^{\prime}} \sum_{t=1}^{T} y_{b, k, t}=1, \quad \forall k \in\{1, \ldots, \delta\} \\
& y_{b, k, t} \in\{0,1\} \quad \forall b \in \beta^{\prime}, \forall k \in\{1, \ldots, n\}, \forall t \in\{1, \ldots, T\}
\end{aligned}
$$

The delivery time of a job $J_{i}$ is given by the addition of the departure time of the batch which contains it and the transportation time between the depot and the location $i(9)$. The other constraints are similar to those used for the previous formulation.

We denote by $c_{b k t}^{\prime}$ the reduced cost of variable $y_{b k t}$. Let $\tilde{\beta}^{\prime} \subseteq \beta^{\prime}$. The subproblem searches for an element of $\beta^{\prime} \backslash \tilde{\beta}^{\prime}$ such that the reduced cost of a new column is negative. 


$$
\begin{aligned}
c_{b k t}^{\prime} \leq 0 \Leftrightarrow \sum_{i=1}^{n} & \underbrace{\left(t+R_{i, b}\right)}_{d_{i}} a_{i, b}+\underbrace{\sum_{i=1}^{n}\left(p_{i, 1}\left(\sum_{k^{\prime}=1}^{n} \sum_{l=k}^{k^{\prime}} \alpha_{l, k^{\prime}}\right)-\gamma_{i}\right)}_{l_{i}^{\prime}} a_{i, b}+ \\
& +P_{b, 2} \underbrace{\left(\sum_{k^{\prime}=k+1}^{n} \sum_{l=1}^{k} \alpha_{l, k^{\prime}}\right)}_{q} \leq \underbrace{\beta_{k}+t \delta_{k}-\sigma_{k-1}+\sigma_{k}+\xi_{k}}_{r_{k t}^{\prime}}
\end{aligned}
$$

where $\alpha_{l k^{\prime}}, \beta_{k}, \gamma_{i}, \delta_{k}, \sigma_{k}$ and $\xi_{k}$ the dual values associated to the constraints (9), (10),(11),(12),(13) and (14) respectively. The dual values $\xi_{k}$ exists only for $k \leq \delta, \sigma_{k-1}$ for $k \geq 2$ and $\sigma_{k}$ for $k \leq n-1$.

An auxiliary graph $G^{\prime}$ is defined in the same way as the one defined above so that the sub-problem consists in finding an elementary shortest path with resource constraints (ESPPRC) on graph $G^{\prime}$ in which the distance $d_{i j}$ of an arc $\left(v_{i}, v_{j}\right)$ is equal to $l_{i}^{\prime}+q \cdot t_{i j}$. However, a new label is introduced in order to store the elapsed time $R_{i b}$ between the departure time of the vehicle and the arrival time to location $i$. To optimally solve the (ESPPRC), Lozano et al algorithm is also used.

\section{Computational Results}

The results are performed on a set of data generated as follows. For each job, processing times and the size follow a sets of discrete uniform distribution $\mathcal{U}(1,100)$ and $\mathcal{U}(1,10)$ respectively. For a location $j$, integer coordinates $\left(X_{j}, Y_{j}\right)$ are randomly generated in the interval $[1,40]$ and the distances between the locations are obtained by computing the classical euclidean distance.

$$
t_{i, j}=t_{j, i}=E\left(\sqrt{\left(X_{i}-X_{j}\right)^{2}+\left(Y_{i}-Y_{j}\right)^{2}}\right)
$$

Note that the processing times, locations and sizes of the jobs are generated independently of each other. Capacity $c$ of the vehicle is fixed to 20 and 5 instances are generated for each number of jobs $n \in\{20,30,40,50,60,70,80,90,100\}$. Hence, the experiments are performed on 45 instances.

The column generation process could be carried out efficiently only for the $D_{\max }$ criterion. Indeed, for the $\sum D_{j}$ model, the need to explicitly represent time makes the convergence much slower as the number of variables becomes huge in the master problem.

The experiments have been implemented for the 45 instances on a Xeon $3.20 \mathrm{GHz}$ computer with 8GB using ILOG CPLEX 12.6 to solve the LPs. We evaluate and compare the solutions obtained by the column generation which represents a lower bound (LB) for the problem with the integer solution obtained by branch and bound on the generated columns, which represents an upper bound (UB). In order to obtain 


\begin{tabular}{c|cccc|}
$n$ & $L B(\mathrm{sec})$ & $\#$ col & $\% G A P_{\text {init }}$ & $\% G A P_{U B}$ \\
\hline 20 & 1.1 & 203 & 21.14 & 4.62 \\
30 & 4.6 & 363 & 13.67 & 1.83 \\
40 & 12.1 & 595 & 9.94 & 0.99 \\
50 & 46.4 & 954 & 10.34 & 0.88 \\
60 & 86.4 & 1136 & 10.21 & 0.69 \\
70 & 215.1 & 1670 & 7.30 & 0.57 \\
80 & 383.7 & 1963 & 7.67 & 0.42 \\
90 & 804.8 & 2637 & 5.44 & 0.39 \\
100 & 1181.9 & 3041 & 5.06 & -
\end{tabular}

Table 1. Computational results for $1 \rightarrow D, k \geq 1|v=1, c| D_{\max }$ problem

an upper bound in a reasonable time, the sub-problem add a single column at each time. Therefore, to obtain a good upper bound, all columns with negative reduced costs are integrated to the master problem which has the advantage of providing good upper bounds and the disadvantage of a larger execution times.

In table 1 , we were interested on the aggregate results for each value of $n$. The statistics take into account the average CPU times for the column generation (column $L B(s e c)$ ). The number of columns generated during the process is given in column \#col. The gap between the initial solution and the relaxed solution is given in column $\left(\% G A P_{\text {init }}\right)$. Finally, the gap between UB solution and the relaxed solution is given by column $\left(\% G A P_{U B}\right)$. The results show a gap lower than $1 \%$ for instances with $n \geq 40$, which proves the very good quality of the bounds. The computational times remain lower than 1200 s for instances with up to 100 jobs.

\section{Conclusions}

In this paper we presented an integrated production scheduling and delivery routing problem that can be seen as a variant of the multi-trip traveling salesman problem with a constrained batching phase due to machine sequencing constraints. We presented complexity results for particular cases and an efficient column generation scheme for the makespan criterion. In the near future, we shall focus on the implementation of a branch-and-price algorithm to close the remaining gap. We will also focus on finding a better decomposition scheme for the sum of deliveries criterion.

\section{ACKNOWLEDGEMENT}

This work was supported by the financial support of the ANR ATHENA project, grant ANR-13-BS02-0006 of the French Agence Nationale de la Recherche.

\section{References}

1. Armstrong, R., Gao, S., Lei, L.: A zero-inventory production and distribution problem with a fixed customer sequence. Annals of Operations Research. 159 (1), 395414. (2008) 
2. Azi, N., Gendreau, M., Potvin, J.Y.: An exact algorithm for a vehicle routing problem with time windows and multiple use of vehicles. European Journal of Operational Research. 202(3), 756-763. (2010)

3. Chang, Y.C., Lee, C.Y.: Machine scheduling with job delivery coordination. European Journal of Operational Research. 158 (2), 470-487 (2004)

4. Chen, Z.L.: Integrated Production and Outbound Distribution Scheduling: Review and Extensions. Operations Research, 58 (1), 130-148. (2010)

5. Chen, B., Lee, C.Y.: Logistics scheduling with batching and transportation. European Journal of Operational Research. 189 (3), 871-876. (2008)

6. Gao, S., Qi, L., Lei, L.: Integrated batch production and distribution scheduling with limited vehicle capacity. International Journal of Production Economics. 160, 13-25. (2015)

7. Garey, M.R., Johnson, D.S., Sethi, R.: The complexity of flowshop and jobshop scheduling. Mathematics of Operations Research. 2 (1), 117-129. (1976)

8. Geismar, H.N., Laporte, G., Lei, L., Sriskandarajah, C.: The Integrated Production and Transportation Scheduling Problem for a Product with a Short Lifespan. Journal on Computing. 20 (1), 21-33. (2008)

9. Johnson, S.M.: Optimal Two-and-Three-Stage Production Schedules with Set-up Times included. Naval Research Logistics. 1 (1), 61-68. (1954)

10. Hall, N.G., Lesaoana, M., Potts, M.C.: Scheduling with Fixed Delivery Dates. Operations Research. 49(1), 134-144. (2004)

11. Lee, C.Y., Chen, Z.L.: Machine scheduling with transportation considerations. Journal of Scheduling. 4 (1), 3-24. (2001)

12. Li, C.L., Ou, J.: Machine scheduling with pickup and delivery. Naval Research Logistics. 52 (7), 617-630. (2005)

13. Li, C.L., Vairaktarakis, G., Lee, C.L.: Machine scheduling with deliveries to multiple customer locations. European Journal of Operational Research. 164 (1), 39-51. (2005)

14. Lozano, L., Duque, D., Medaglia, A.L.: An Exact Algorithm for the Elementary Shortest Path Problem with Resource Constraints. Transportation Science. (2015)

15. Lysgaard, J., Sanne W.: A branch-and-cut-and-price algorithm for the cumulative capacitated vehicle routing problem. European Journal of Operational Research. 236(3), 800-810. (2014)

16. Viergutz, C., Knust, S.: Integrated production and distribution scheduling with lifespan constraints. Annals of Operations Research. 213 (1), 293-318. (2014)

17. Wang, X., Cheng, T.C.E.: Production scheduling with supply and delivery considerations to minimize the makespan. European Journal of Operational Research. 194 (3), 743-752. (2009) 